Editorial

\title{
Screening for breast cancer in the Eastern Mediterranean Region
}

\author{
A.B. Miller ${ }^{7}$
}

Breast cancer is the most important cancer in women in the World Health Organization (WHO) Eastern Mediterranean Region (EMR), being the number one cancer in 16 of the 22 countries. In many technicallyadvanced countries mammography screening, generally from the age of 50 years, is the most common breast cancer control strategy performed. The WHO has recommended a step-wise approach to introducing screening, beginning with the promotion of early diagnosis through public and professional education, to pilot studies of organized programmes, followed by national programmes as resources permit $[1,2]$. This approach has been incorporated in the Cancer Control Strategy of the EMR Office of WHO. In summary, the planning steps include:

\section{1: Where are we now?}

- Assess the cancer problem;

- Assess the evidence-base for screening;

- Consider alternative strategies to reach the same objectives (primary prevention or improved treatment);

- Assess the existing early detection plan and ongoing activities

\section{2: Where do we want to be?}

- Define the target population for early detection of frequent cancers;

- Identify gaps in early detection services;

- Set objectives for early diagnosis and screening;
- Assess the feasibility of screening interventions;

- Set priorities for screening.

\section{3: How do we get there?}

- Plan procurement of key resources;

- Determine activities for early diagnosis and screening;

- Work with multidisciplinary and multisectoral teams;

- Move from policy to implementation.

Working through these steps in relation to each priority cancer site provides the basis for the organization of screening and enables strategic decisions to be made to start or stop screening for a specific site as part of a country's $\mathrm{Na}$ tional Cancer Control Plan.

In this issue of the EMHJ, Akhtar et al. describe the initial results from the pilot phase of a mammography screening programme in Saudi Arabia. The authors are to be complimented on conducting this pilot phase in a defined area. A great deal of care seems to have been taken to try and ensure the project was a success. However, compliance with attendance for mammography screening was low, and the rate of recall for abnormalities suspected high, while the numbers of breast cancers detected were small. The authors admit that by the standards of many organizations in the more developed countries, various indicators suggest a need for much more attention to some of the components of an organized screening programme.
In the study of Akhtar et al., 4 women were detected with invasive breast cancer as a result of the screening of 1628 women, a detection rate of 2.5 per 1000. This breast cancer detection rate was low, largely because the majority of those screened were young. The age of 1 woman detected with breast cancer was unknown, but of the 3 with known ages, all were between 41 and 50 years. The 1 case of ductal carcinoma in situ (DCIS) was detected in a younger woman. However, there is no good evidence that detecting cases of DCIS is beneficial as these lesions, though markers of risk, are not precursors of breast cancer in the classic sense.

The numbers of detected invasive cancers, though small, are compatible with the expected incidence of breast cancer in Saudi Arabia in women aged 40-49 years. They are lower, however, than was found in a population-based breast screening trial in Cairo, Egypt, which used clinical breast examinations performed by specially trained female doctors in sessions at primary health centres as the primary screen, mammography being used for diagnosis in a hospital serving the community [3]. Possible explanations for the higher detection rate in Cairo are that the social workers, who visited women in their homes to invite them for screening, were more successful in persuading women with potential breast problems to attend, and breast cancer incidence is higher in Egypt than in many other countries in the EMR, including Saudi Arabia. Thus the Cairo trial has shown 
that it is feasible to use social workers to invite women to attend for screening breast examinations while there is preliminary indication that a stage shift has been achieved [4]. In Morocco, after a pilot study of mammography screening, it has been decided to base breast screening nationally on clinical breast examinations. A similar decision has been taken in Oman. In Egypt, the Ministry of Health and Population has initiated a mammography screening programme, using digital mammography, with images read in a central location. Although based on mobile units visiting primary health care centres, this seems to be largely opportunistic. Data on coverage of the target group have not been published.

Successful screening requires a fully organized programme. An organized screening programme has a number of components, including:

- An identifiable target group or population, with accompanying population registers;

- Implementation measures available to guarantee high coverage and participation;

- Access to high-quality screening;

- Effective referral system in place for diagnosis and treatment;

- Measures in place to monitor and evaluate a programme [5].

The alternative to organized screening is opportunistic, or laissez faire, screening. Organized screening is distinguished from opportunistic screening primarily on the basis of how invitations to screening are extended. In organized screening, invitations should be issued to those at risk in a defined target population, preferably through population registers, and measures instituted to facilitate their attendance for screening. In this context, risk is usually defined by age and sex. In opportunistic screening, invitations to screening are extended to individuals when they encounter health care providers for reasons unrelated to cancer. Opportunistic screening is often inefficient because many who are screened are not at high risk of cancer; many in the population who should be screened are not, and those that do receive screening may be screened either too frequently or too infrequently. All screening programmes require some degree of organization to be successful, and as the extent of the organization of the various elements of screening increases, so too does the impact of the programme. In the project described by Akhtar et al. the invitations issued were disseminated by public information campaigns, and were not based upon a defined register. It would seem that in this region of Saudi Arabia, this approach was not sufficient to ensure that the majority of women in the target group attended for screening. This suggests that further pilot phases are needed evaluating ways to recruit women into screening before a national programme can be mounted. One option would be to evaluate the use of social workers, as is being done in Sana'a, Yemen, and Khartoum, Sudan, on the lines of the Cairo trial [4].

Another aspect of the study of Akhtar et al. to be reconsidered is the age group of women included. In western Europe and North America, the large majority of programmes do not include women under the age of 50 years. Although this has not been the case in the United States of America (USA), where the American Cancer Society and the American College of Radiology urge annual screening from the age of 40 years, recently the US Preventive Services Task Force recommended that women aged 40-49 years should not receive routine mammography screening [6]. The reasons for this were that the expected benefit was small, and the harm (in the form of excessive investigations) high. The number needed to invite for screening to prevent 1 breast cancer death was calculated as 1904 [7]. I have calculated that in nearly every country in the Region, it would be necessary to screen over 2000 women aged 35-39 years to detect 1 case of breast cancer, approximately double the number that would need to be invited if they were aged 40-49 years, which implies that it would be necessary to invite approximately 5000 women aged 35-39 years to prevent 1 death from breast cancer under the assumption that there is a benefit from screening in this age group, for which there is, as yet, no evidence. Thus, it would be more efficient in the future not to attempt to recruit women under the age of 40 years. Indeed including women from the age of 35 years in routine mammography screening programmes has never been advocated in the more developed countries, even in the USA.

Because of the larger number of younger than older women in most countries in the EMR, clinically a higher proportion of women in their thirties and forties present with breast cancer than would be the expectation in western Europe and North America. This has led to the misunderstanding that somehow breast cancer is different in the countries in the Region than in more developed countries. However, when population-based cancer registries are active and it is possible to compute the risk of the development of breast cancer by age, it appears that the incidence of breast cancer at each age is no higher, though it is often much lower at older ages. This creates a problem for countries in the Region considering mammography screening, as it is now accepted that mammography screening is less effective in women under the age of 50 years than in older women $[7,8]$.

The difficulty in recruiting women into screening, and its likely low efficacy in relation to the main burden of breast cancer in younger women, suggests that there is a substantial risk of very little benefit from breast screening in the Region, and that the priority should shift to early diagnosis through health education, combined with effective treatment of detected cases. 
Early diagnosis is defined by WHO as the awareness (by the public or health professionals) of early signs and symptoms of cancer in order to facilitate diagnosis before the disease becomes advanced [2]. This enables more effective and simpler therapy. The concept of early diagnosis is sometimes called "down-staging". In a population where the majority of the cancers amenable to early detection are diagnosed in late stages, the establishment of an early diagnosis programme may be the most feasible strategy to reduce the percentage of advanced stages and improve survival rates [2]. To introduce an early diagnosis programme based on health education, however, will require special studies to help understand women's' concept of the importance of breast cancer and its curability if detected early, and the ways to change often basic misunderstandings. Any education programme devised must be culturally appropriate, so it cannot simply be imported from the types of programmes and pamphlets developed in other regions. Further, it may be necessary to ensure such education is also addressed to men as they may be influential in the decision-making that results in a woman attending a primary health care centre for diagnosis if she becomes concerned about her breasts. It will also mean that primary care physicians may have to undergo re-training, partly to ensure they do not have the same misunderstanding of the potential curability of breast cancer as their patients, but also so that they are able to recognize the signs of early breast cancer.

Once an early diagnosis programme is in place and there is evidence that it is being successful, then, in accordance with WHO guidelines, it would be appropriate to consider screening. However, if resources are not sufficient to consider nationwide mammography screening, it might be preferable to start by introducing pilot programmes of Clinical Breast Examination screening, along the lines of the Cairo trial, or following the model of Morocco and Oman, reserving mammography for diagnosis. It is relevant that in the Canadian National Breast Screening Study we found no benefit from adding annual mammography to clinical breast examination screening in women aged 50-59 years [9].

\section{References}

1. WHO Guide for Effective Programmes. Cancer control. Knowledge into action: planning. Geneva, World Health Organization, 2006.

2. WHO Guide for Effective Programmes. Cancer control. Knowledge into action: Early detection. Geneva, World Health Organization, 2007.

3. Boulos $\mathrm{S}$ et al. Breast screening in the emerging world: high prevalence of breast cancer in Cairo. The Breast, 2005, 14:340-346.

4. Miller A. Practical applications for clinical breast examination (CBE) and breast self-examination (BSE) in screening and early detection of breast cancer. Breast Care, 2008, 3:17-20.

5. Hakama $\mathrm{M}$ et al. Evaluation of screening programmes for gynecological cancer. British Journal of Cancer, 1985, 52:669-73.
6. US Preventive Services Task Force. Screening for breast cancer: U.S. Preventive Services Task Force recommendation statement. Annals of Internal Medicine, 2009,151:716-726.

7. Nelson HD et al. Screening for breast cancer: an update for the U.S. Preventive Services Task Force. Annals of Internal Medicine , 2009, 151:727-737.

8. IARC Handbooks on cancer prevention, vol 7. Breast cancer screening. Lyon, IARC Press, 2002.

9. Miller AB et al. Canadian National Breast Screening Study - 2.: 13-year results of a randomized trial in women aged 50-59 years. Journal of the National Cancer Institute, 2000, 92:1490-1499. 